\title{
Reconstructing Lanchester Warfare Models Through a Lagrangian Mechanics Lens
}

\author{
J. Gill $^{\mathrm{a}}{ }^{\text {, A. Gill }}{ }^{\mathrm{b}}$ (D) and J. Penfold ${ }^{\mathrm{C}}$

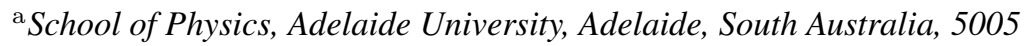 \\ b JOAD, Defence Science and Technology Group, Edinburgh, South Australia, 5111 \\ ' JOAD, Defence Science and Technology Group, Edinburgh, South Australia, 5111 \\ Email: joshua.gill@student.adelaide.edu.au
}

\begin{abstract}
Warfare modelling is an important component of research in Defence. Even with the rise in performance of modern-day computers, simulating war is not only time-consuming but potentially obfuscates the determining factors in victory. A desirable goal of investigating warfare models is to predict the minimum force ratio required to ensure victory for one side, so-called victory conditions. Denoting the following model:

$$
\dot{x}=-a(t) y, \quad \dot{y}=-b(t) p(t, y) x, \quad x(t=0)=x_{0}, \quad y(t=0)=y_{0}
$$

as the time-dependent Generalised Ambush model, where $a(t)$ and $b(t)$ are weapon attrition rates and $p(t, y)$ determines the firing behaviour of $x$, Kress \& MacKay (2013) studied the autonomous (time-independent) case $(a(t)=a, b(t)=b, p(t, y)=p(y))$ and derived the victory condition through the classical Lanchester (conservation) law approach. Taylor (1978, 1981) and Taylor \& Brown (1983) extensively analysed the nonautonomous (time-dependent) version of Lanchester's Modern Warfare model $(p(t, y)=1)$, however, the conservation law approach was abandoned in favour of solving for the force trajectories explicitly.
\end{abstract}

This paper investigates the idea that Lanchester warfare models could be equivalently expressed as Lagrangian mechanics problems, where the underlying symmetries might be exploited. The motivation for doing so is the observation that time-dependent conservation laws exist in such systems. In particular, a Noether symmetry will be sought to determine a conserved quantity of the system from which the victory condition can be deduced.

To recast in Lagrangian mechanics requires solving the inverse problem, that is, in finding a Lagrangian that equivalently represents the Lanchester system of equations. Fortunately, the method of Darboux (1878) provides an algorithmic procedure to compute the Lagrangian $L(t, x, \dot{x})$ associated with (1) via integration. From the various types of symmetries examined by Mei et al. (2014), the Noether symmetry was examined here, which requires finding a solution to Noether's Identity in terms of infinitesimal generators and a gauge function. If $\exists \xi_{0}(t, x, \dot{x}), \xi(t, x, \dot{x})$ and $G(t, x, \dot{x})$ s.t. (2a) is true, then (2) is the corresponding conserved quantity:

$$
\dot{\xi_{0}} L+\xi_{0} \frac{\partial L}{\partial t}+\xi \frac{\partial L}{\partial x}+\left(\dot{\xi}-\dot{x} \dot{\xi_{0}}\right) \frac{\partial L}{\partial \dot{x}}+\dot{G}=0, Q(t, x, \dot{x})=L \xi_{0}+\frac{\partial L}{\partial \dot{x}}\left(\xi-\dot{x} \xi_{0}\right)+G
$$

For Lanchester's classic modern warfare model, we demonstrate the existence of a time-dependent conservation law and conserved quantity and illustrate graphically how it reproduces the more familiar square law and force trajectory results. For two non-autonomous versions of (1), we also derive the associated Lagrangian, the form of the conserved quantity and demonstrate when $a(t) \propto b(t)$ how the Lagrangian formulation returns the equivalent victory conditions as deduced via separation of variables.

We hope that this initial exploration of reconstructing Lanchester warfare models as equivalent Lagrangian mechanics problems, and the associated search for symmetries and conserved quantities, may open up a fruitful path towards deriving analytic victory conditions for a range of time-dependent models.

Keywords: Lanchester model, Lagrangian mechanics, Noether symmetry, conserved quantity 
J. Gill et al., Reconstructing Lanchester Warfare Models ...

\section{INTRODUCTION}

\subsection{Background}

Warfare modelling is an important component of research in Defence. Even with the power of modern-day computers, simulating war is not only time-consuming but potentially obfuscates the determining factors in victory. One goal of investigating models of warfare is to predict the minimum force ratio $\left(u_{0}=x_{0} / y_{0}\right.$, $x$-friendlies, $y$-enemies) for victory, namely, victory conditions. The models investigated here stem from an autonomous generalised ambush model, well-documented by Kress \& MacKay (2013):

$$
\dot{x}(t)=-a y(t), \quad \dot{y}(t)=-b p(y) x(t), \quad x(t=0)=x_{0}, \quad y(t=0)=y_{0}
$$

where the weapon attrition rates are $a$ and $b$ and the function $p(y)$ controls the firing behaviour of $x$ (i.e. $p(y)=1$ gives Lanchester's Modern Warfare, while $p(y)=y$ gives Deitchman's Guerrilla Warfare Deitchman (1962)).

The standard solution method to these problems is to 'divide' out the explicit time component. By taking the ratio of the derivatives, separating the variables and integrating:

$$
\frac{\dot{x}}{\dot{y}}=\frac{d x}{d t} \frac{d t}{d y}=\frac{d x}{d y}=\frac{a y(t)}{b p(y) x(t)} \Longrightarrow b \int_{x_{0}}^{x} v d v=a \int_{y_{0}}^{y} \frac{u d u}{p(u)}
$$

leads to:

$$
b x^{2}(t)-2 a F(y(t))=b x_{0}^{2}-2 a F\left(y_{0}\right), \forall t \geq 0, \text { where } F(y)=\int_{0}^{y} \frac{u d u}{p(u)} .
$$

Since the left-hand side holds for $x$ and $y$ at any time, we refer to (5) as a Lanchester conservation law. For an $x$ victory, $\exists t=t_{F}<\infty$ such that $y\left(t_{F}\right)=y_{F}=0$ and $x\left(t_{F}\right)=x_{F}>0$. Applying this to (5) leads to the victory condition:

$$
\frac{x_{0}}{y_{0}}=u_{0}>\sqrt{\frac{2 a F\left(y_{0}\right)}{b y_{0}^{2}}} .
$$

Some common choices for $p(y)$ lead to the following well known conservation laws and associated victory conditions:

$$
\begin{gathered}
\text { Lanchester's Modern Warfare: } p(y)=1 \Longrightarrow F(y)=\frac{y^{2}}{2}, \\
\Longrightarrow b x^{2}-a y^{2}=b x_{0}^{2}-a y_{0}^{2} \Longrightarrow \text { Square Law } \Longrightarrow u_{0}>\sqrt{\frac{a}{b}} \\
\text { Deitchman's Guerrilla Warfare: } p(y)=y \Longrightarrow F(y)=y \\
\Longrightarrow b x^{2}-2 a y=b x_{0}^{2}-2 a y_{0} \Longrightarrow \text { Mixed Law } \Longrightarrow u_{0}>\sqrt{\frac{2 a}{b y_{0}}} .
\end{gathered}
$$

A lower bound on the victory conditions is uniquely determined for any choice of $p(y)$. However, where the integral $F(y)$ is not analytic, numeric and/or series expansion give an estimate on the lower bound (see Pincombe et al. (2016) for an example).

\subsection{Motivation}

Extending (3) to include time-dependence, either in the weapon attrition rates or firing behaviour function, would provide a much richer modelling framework. For example, Taylor (1978, 1981) and Taylor \& Brown (1983) have analysed in great detail the time-dependent Lanchester Modern Warfare model but focussed more on solving for the force trajectories than seeking a conservation law. However, conserved quantities and conservation laws are commonplace in mechanics and physics and are not necessarily restricted to autonomous varieties. In this paper, we investigate the novel idea that time-dependent Lanchester warfare models be conceived as Lagrangian mechanics systems, whose underlying symmetries, particularly the Noether symmetry, may admit possibly time-dependent conserved quantities and consequently a Lanchester conservation law. 


\subsection{Scope}

Section 2 will describe how to determine a Lagrangian which equivalently represents the system of differential equations governing Lanchester models. Section 3 will apply that method to two different warfare models. Section 3.1 refers to Lanchester's Autonomous Modern Warfare model and illustrates the effectiveness of Lagrangian mechanics to determine conserved quantities, in particular, explicitly time-dependent conserved quantities. Sections 3.2 and 3.3 look at determining a Lagrangian for non-autonomous extensions of some classic Lanchester warfare models and reducing the condition for symmetry and implicit conserved quantity.

\section{Lagrangian Mechanics Approach}

We consider reconstructing Lanchester warfare models as mechanical systems which exhibit symmetry, leading to conserved quantities. The particular type of mechanical system used here will be Lagrangian mechanics, whereby the Euler-Lagrange equations on some Lagrangian $L$ are an equivalent representation to the system of differential equations. The first task then is to determine an appropriate Lagrangian.

\subsection{Determining the Lagrangian - The method of Darboux}

For any system of two coupled first-order ordinary differential equations (ODEs), they can be reconstructed into a second order ODE (here in $x$, but equivalently in $y$ if the problem permits) as $\ddot{x}+P(t, x, \dot{x})=0$. The Lagrangian $L$ of this system must recover this second order ODE from the Euler-Lagrange equations:

$$
\frac{d}{d t} \frac{\partial L}{\partial \dot{x}}-\frac{\partial L}{\partial x} \equiv \Lambda(t, x, \dot{x})(\ddot{x}+P(t, x, \dot{x})), \quad \Lambda(t, x, \dot{x})=\exp \left(\int \frac{\partial P}{\partial \dot{x}} d t\right),
$$

where $\Lambda(t, x, \dot{x})$ is Jacobi's Last Multiplier (Casetta 2015). The integral in (8) is indefinite but does not require a constant of integration (as it is lost in the calculation of the conserved quantity). As per the method of Darboux (1878), the Lagrangian for this system is:

$$
L(t, x, \dot{x})=\int_{C_{1}}^{\dot{x}}(\dot{x}-\Omega) \Lambda(t, x, \Omega) d \Omega-\int_{C_{2}}^{x} P\left(t, \phi, C_{1}\right) \Lambda\left(t, \phi, C_{1}\right) d \phi,
$$

where $C_{1}$ and $C_{2}$ are freely chosen constants. As discussed by Yan (1978) and Leubnert \& Krumm (1990), they are generally set to zero for simplicity, however, some models require a non-zero choice to derive a Lagrangian.

\subsection{Noether Symmetry}

Assuming a Lagrangian for the system exists, the Lanchester warfare model can now be equivalently represented by a mechanical system with Lagrangian $L$. As in Lagrangian mechanics, symmetry in the model may exist, exhibiting a conservation law and a conserved quantity by extension. As per Mei et al. (2014), a Noether symmetry can be represented mathematically in the form of Noether's Identity, shown in (10 1$)$. Once suitable $\xi_{0}(t, x, \dot{x}), \xi(t, x, \dot{x})$ (the infinitesimal generators of $t$ and $x$ ) and $G(t, x, \dot{x})$ (a gauge function) are found, a (Noether) conserved quantity can be calculated by (10):

$$
\dot{\xi_{0}} L+\xi_{0} \frac{\partial L}{\partial t}+\xi \frac{\partial L}{\partial x}+\left(\dot{\xi}-\dot{x} \dot{\xi_{0}}\right) \frac{\partial L}{\partial \dot{x}}+\dot{G}=0, \quad Q(t, x, \dot{x})=L \xi_{0}+\frac{\partial L}{\partial \dot{x}}\left(\xi-\dot{x} \xi_{0}\right)+G
$$

\section{Using Lagrangian Mechanics}

In theory, reconstructing Lanchester warfare models as a Lagrangian mechanics problem can potentially demonstrate symmetry, leading to a conserved quantity. However, that is reliant on the choice of the infinitesimal generators and gauge function. Moreover, the choice of these could lead to different conserved quantities, although, under the confines of the model, some may be non-informative. A test for this new method shows that a well-known model returns the same victory conditions as originally found. The simplest of models to test this on is the Autonomous Lanchester Modern Warfare Model. Extensions of classic models will also be analysed and the condition leading to symmetry provided.

\subsection{Autonomous Lanchester Modern Warfare}

Consider Lanchester's Autonomous Modern Warfare model:

$$
\dot{x}(t)=-a y(t), \quad \dot{y}(t)=-b x(t), \quad x(t=0)=x_{0}, \quad y(t=0)=y_{0},
$$


J. Gill et al., Reconstructing Lanchester Warfare Models ...

where $a, b$ are constant. As a second order differential equation, this becomes $\ddot{x}-a b x=0$, so $P(t, x, \dot{x})=$ $-a b x$. The Jacobi Last Multiplier, conveniently, for this system, evaluates to $\Lambda(t, x, \dot{x})=1$, as $\frac{\partial P}{\partial \dot{x}}=0$. Following the method of Darboux, the Lagrangian is determined from:

$$
L=\int_{0}^{\dot{x}}(\dot{x}-\Omega) d \Omega+a b \int_{0}^{x} \phi d \phi=\frac{1}{2}\left(\dot{x}^{2}+a b x^{2}\right) .
$$

Now that a Lagrangian has been determined, this is used to find the infinitesimal generators and gauge function which satisfy Noether's Identity ((10)). Substituting (12) into (10^) and (10) (recalling that $\dot{x}=-a y)$, they reduce to:

$$
\frac{\dot{\xi_{0}}}{2}\left(a b x^{2}-\dot{x}^{2}\right)+\dot{x} \dot{\xi}+a b x \xi+\dot{G}=0, \quad Q(t, x, y)=\frac{a}{2}\left(b x^{2}-a y^{2}\right) \xi_{0}-a y \xi+G .
$$

Note that the choice $\xi_{0}=1 / a$ and $\xi=G=0$ satisfies Noether's Identity, but gives the same time-independent conserved quantity found via separation of variables $((7 b))$. However, if we choose $\xi_{0}=0, \xi=\exp (-A t)$ and $G=A x \exp (-A t)$, for some constant $A$, substituting into the Noether Identity ((13a)) yields $(a b-$ $\left.A^{2}\right) x \exp (-A t)=0$. Hence, the Noether Identity is satisfied at all time only when $A= \pm \sqrt{a b}$. Updating the infinitesimal generators and gauge function and substituting into the conserved quantity ((13b)) gives:

$$
Q(t, x, y)=( \pm \sqrt{a b}) \exp (\mp t \sqrt{a b})\left(x \mp y \sqrt{\frac{a}{b}}\right)
$$

This demonstrates the existence of an explicitly time-dependent conserved quantity. Equating the conserved quantity at both end points of the battle $Q\left(t=0, x_{0}, y_{0}\right)=Q\left(t=t_{F}, x_{F}, y_{F}=0\right)$, and imposing $x_{F}>0$, the known victory conditions is easily recovered:

$$
\begin{aligned}
& x_{F}=\exp \left( \pm t_{F} \sqrt{a b}\right)\left(x_{0} \mp y_{0} \sqrt{\frac{a}{b}}\right)>0 \\
& \Longrightarrow x_{0} \mp y_{0} \sqrt{\frac{a}{b}}>0, \quad \text { so that } \quad \frac{x_{0}}{y_{0}}=u_{0}> \pm \sqrt{\frac{a}{b}} .
\end{aligned}
$$

Noting that the negative case gives a non-informative victory condition, the only sensible option is the positive case, equivalent to that deduced by Lanchester via separation of variables.

Although only the positive case gives an informative victory condition, both conserved quantities are technically valid, so additional conserved quantities can be found by taking suitable combinations for any set of conserved quantities. In particular, by taking the product of those conserved quantities in (15):

$$
\begin{aligned}
Q(t, x, y) & =(\sqrt{a b}) \exp (-t \sqrt{a b})\left(x-y \sqrt{\frac{a}{b}}\right)(-\sqrt{a b}) \exp (t \sqrt{a b})\left(x+y \sqrt{\frac{a}{b}}\right) \\
& =-a b\left(x^{2}-\frac{a y^{2}}{b}\right) \equiv b x^{2}-a y^{2}
\end{aligned}
$$

and discarding the $-a$ factor without loss of generality, recovers the time-independent conserved quantity derived via separation of variables.

Whilst the Lagrangian method is more involved, it does provide an alternative conserved quantity leading to equivalent victory conditions. Additionally, as shown in Figure 1, the time-dependent conserved quantity recovers the time-independent conserved quantity and both force trajectories via suitable projections onto each two-dimensional plane.

\subsection{Non-autonomous Lanchester Modern Warfare}

Consider now time-dependent attrition rates, $a(t)$ and $b(t)$, such that the system of equations become:

$$
\dot{x}=-a(t) y, \quad \dot{y}=-b(t) x, \quad x(t=0)=x_{0}, \quad y(t=0)=y_{0} .
$$

The method of Darboux can be applied here to determine the Lagrangian of the system. Reducing (17) to:

$$
\ddot{x}-\dot{x} \frac{\dot{a}(t)}{a(t)}-a(t) b(t) x=0, \quad \text { where } P(t, x, \dot{x})=-\dot{x} \frac{\dot{a}(t)}{a(t)}-a(t) b(t) x,
$$


J. Gill et al., Reconstructing Lanchester Warfare Models ...

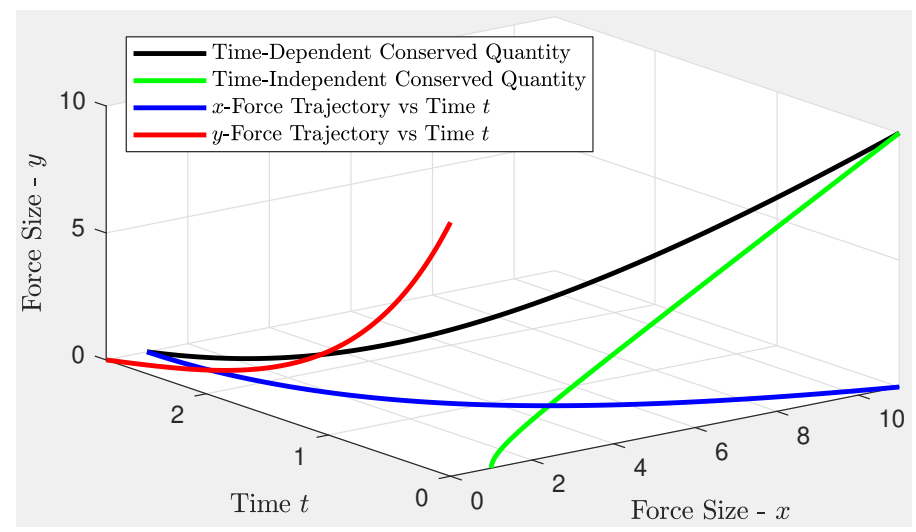

Figure 1. Time-Dependent Conserved Quantity: Relation to Force Trajectories and Lanchester's Square Law

Jacobi's Last Multiplier becomes:

$$
\Lambda(t, x, \dot{x})=\exp \left(\int-\frac{\dot{a}(t)}{a(t)} d t\right)=\frac{1}{a(t)} .
$$

The Lagrangian can now be determined by the method of Darboux:

$$
L=\frac{1}{a(t)} \int_{0}^{\dot{x}}(\dot{x}-\Omega) d \Omega+b(t) \int_{0}^{x} \phi d \phi=\frac{1}{2}\left(\frac{\dot{x}^{2}}{a(t)}+b(t) x^{2}\right) .
$$

Substituting into (10 ) (Noether Identity) and grouping powers of $x$ and $\dot{x}$ results in:

$$
\begin{aligned}
& -\frac{\dot{x}^{2} \xi_{0}}{2 a(t)}\left(\frac{\dot{\xi}_{0}}{\xi_{0}}+\frac{\dot{a}(t)}{a(t)}\right)+\frac{x^{2}}{2}\left(b(t) \dot{\xi}_{0}+\xi_{0} \dot{b}(t)\right)+\dot{x} \frac{\dot{\xi}}{a(t)}+x b(t) \xi+\dot{G}=0 \\
\Longrightarrow & -\frac{\dot{x}^{2} \xi_{0}}{2 a(t)}\left[\frac{d}{d t}\left(\ln \left(a(t) \xi_{0}\right)\right)\right]+\frac{x^{2}}{2}\left[\frac{d}{d t}\left(b(t) \xi_{0}\right)\right]-y \dot{\xi}-\dot{y} \xi+\dot{G}=0, \quad\left(y=-\frac{\dot{x}}{a(t)}, \dot{y}=-b(t) x\right) \\
\Longrightarrow & \frac{\dot{x}^{2} \xi_{0}}{2 a(t)}\left[\frac{d}{d t}\left(\ln \left(\frac{1}{a(t) \xi_{0}}\right)\right)\right]+\frac{x^{2}}{2}\left[\frac{d}{d t}\left(b(t) \xi_{0}\right)\right]+\frac{d}{d t}(G-y \xi)=0 \\
\Longrightarrow & \frac{\dot{x}^{2} \xi_{0}}{2 a(t)}\left[\frac{d}{d t}\left(\ln \left(\frac{1}{a(t) \xi_{0}}\right)\right)\right]+\frac{x^{2}}{2}\left[\frac{d}{d t}\left(b(t) \xi_{0}\right)\right]+\frac{d}{d t}\left(G+\xi \frac{\dot{x}}{a(t)}\right)=0 .
\end{aligned}
$$

The difficulty now arises from the choice of the infinitesimal generators and the gauge function. A sufficient, but not necessary, condition for Noether's Identity to hold is for each term above to individually be zero. For the first and second terms, this requires $\xi_{0} \propto 1 / a(t)$ and $\xi_{0} \propto 1 / b(t)$, hence, is only satisfied for proportional attrition rates. The third term is always satisfied for $\xi \propto a(t) / \dot{x}$ and $G$ constant. For the special case where $a(t) \propto b(t), \xi_{0}=1 / a(t), \xi=a(t) / \dot{x}$ and $G=-1$, and substituting into (10 $)$ ), the following conserved quantity for this system is found (recalling $\dot{x}=-a(t) y$ ):

$$
\begin{aligned}
Q(t, x, y) & =\frac{1}{2}\left(b(t) x^{2}-a(t) y^{2}\right) \xi_{0}-y \xi+G \\
& =\frac{1}{2}\left(x^{2} \frac{b(t)}{a(t)}-y^{2}\right) \quad\left(\text { since } \xi=\frac{a(t)}{\dot{x}}=-\frac{1}{y}, \text { and } G=-1\right) .
\end{aligned}
$$

This results in the same victory condition as found for the autonomous case, due to the proportional timedependence of the attrition rates. This assumption of proportionality appears to provide the symmetry required for Noether's Identity to hold. Potentially other constraints on the attrition rates, $a(t)$ and $b(t)$ may provide alternative symmetries for this model. Trivially, if $a(t)=a$ and $b(t)=b$, the choice of $\xi_{0}=2$ and $\xi=G=0$ recovers the conserved quantity and victory condition found via separation of variables for the Autonomous Lanchester Modern Warfare model. 
J. Gill et al., Reconstructing Lanchester Warfare Models ...

\subsection{Non-autonomous Deitchman Guerrilla Warfare}

Consider now time-dependent attrition rates, $a(t)$ and $b(t)$, on the Deitchman Guerrilla Warfare, such that the system of equations become:

$$
\dot{x}=-a(t) y, \quad \dot{y}=-b(t) x y, \quad x(t=0)=x_{0}, \quad y(t=0)=y_{0} .
$$

The method of Darboux can also be applied here to determine the Lagrangian of the system. The only difference here is that the system of differential equations is reduced to a second-order differential equation in $y$ :

$$
\ddot{y}-\dot{y} \frac{\dot{b}(t)}{b(t)}-a(t) b(t) y^{2}-\frac{\dot{y}^{2}}{y}=0, \quad \text { where } P(t, y, \dot{y})=-\dot{y} \frac{\dot{b}(t)}{b(t)}-a(t) b(t) y^{2}-\frac{\dot{y}^{2}}{y},
$$

so that Jacobi's Last Multiplier becomes:

$$
\Lambda(t, y, \dot{y})=\exp \left(\int\left[-\frac{\dot{b}(t)}{b(t)}-2 \frac{\dot{y}}{y}\right] d t\right)=\frac{1}{b(t) y^{2}} .
$$

The Lagrangian can now be determined by the method of Darboux as:

$$
L=\frac{1}{b(t) y^{2}} \int_{0}^{\dot{y}}(\dot{y}-\Omega) d \Omega+a(t) \int_{0}^{y} d \phi=\frac{1}{2 b(t)} \frac{\dot{y}^{2}}{y^{2}}+a(t) y .
$$

Substituting this Lagrangian into (10a) (Noether's Identity) and grouping like infinitesimal generators, (recalling $\dot{x}=-a(t) y, \dot{y}=-b(t) x y$ ), yields the following condition:

$$
\begin{aligned}
& \dot{\xi_{0}}\left(a(t) y-\frac{1}{2 b(t)} \frac{\dot{y}^{2}}{y^{2}}\right)+\xi_{0}\left(-\frac{\dot{b}(t)}{2 b^{2}(t)} \frac{\dot{y}^{2}}{y^{2}}+\dot{a}(t) y\right)+\xi\left(-\frac{1}{b(t)} \frac{\dot{y}^{2}}{y^{3}}+a(t)\right)+\frac{\dot{\xi}}{b(t)} \frac{\dot{y}}{y^{2}}+\dot{G}=0 \\
& \Longrightarrow-\frac{\xi_{0}}{2 b(t)} \frac{\dot{y}^{2}}{y^{2}}\left(\frac{\dot{\xi_{0}}}{\xi_{0}}+\frac{\dot{b}(t)}{b}\right)+y\left(a(t) \dot{\xi_{0}}+\dot{a}(t) \xi_{0}\right)-\xi\left(\frac{\dot{x}}{y}-\frac{x \dot{y}}{y^{2}}\right)-\dot{\xi}\left(\frac{x}{y}\right)+\dot{G}=0 \\
& \Longrightarrow \frac{\xi_{0}}{2 b(t)} \frac{\dot{y}^{2}}{y^{2}}\left[\frac{d}{d t}\left(\ln \frac{1}{b(t) \xi_{0}}\right)\right]+y\left[\frac{d}{d t}\left(a(t) \xi_{0}\right)\right]+\left[\frac{d}{d t}\left(G-\xi\left(\frac{x}{y}\right)\right)\right]=0 \\
& \Longrightarrow \frac{\xi_{0}}{2 b(t)} \frac{\dot{y}^{2}}{y^{2}}\left[\frac{d}{d t}\left(\ln \frac{1}{b(t) \xi_{0}}\right)\right]+y\left[\frac{d}{d t}\left(a(t) \xi_{0}\right)\right]+\frac{d}{d t}\left[G+\xi \frac{\dot{y}}{b(t) y^{2}}\right]=0 .
\end{aligned}
$$

The difficulty again is the choice of the infinitesimal generators and the gauge function. Applying the sufficient, but not necessary, condition that each term is zero and assuming that $a(t) \propto b(t)$, a solution exists for $\xi_{0}=$ $1 / a(t), \xi=b(t) y^{2} / \dot{y}$ and $G=-1$. Substituting into (10) and recalling $\dot{y}=-b(t) x y$, the following conserved quantity for this system is found:

$$
Q(t, x, y)=\left(a(t) y-\frac{1}{2} b(t) x^{2}\right) \xi_{0}-\frac{\xi x}{b(t) y}+G=y-\frac{x^{2}}{2} \frac{b(t)}{a(t)}
$$

which returns the same victory condition as obtained by separation of variables. Another combination of the infinitesimal generators or gauge function might provide a unique conserved quantity or limit the constraint imposed on the attrition rates here. Trivially, for $a(t)=a$ and $b(t)=b$, the victory condition for the Autonomous Ambush Model is recovered for $\xi_{0}=1, \xi=G=0$.

\section{DISCUSSION}

By reconstructing Lanchester warfare models through the lens of Lagrangian mechanics, symmetries of the dynamics can be exposed. By the method of Darboux (1878) and extending on work done by Mei et al. (2014), the Lagrangian, Noether Identity and corresponding conserved quantity can all be expressed for Lanchester warfare models. If suitable choices of the infinitesimal generators and gauge function imply that Noether's Identity holds, the corresponding conserved quantity provides a path to analytic victory conditions. It has been shown that the application of the Lagrangian approach to the autonomous Lanchester's Modern Warfare model yields identical victory conditions via a unique, time-dependent conserved quantity. Furthermore, in the particular case of proportionality between the attrition rates, the time-dependent extension of Lanchester's Modern Warfare model also yields an analytic victory condition. While a general solution to the non-autonomous extension of Lanchester's Modern Warfare model is not found here, the Lagrangian mechanical lens provides a novel way to analyse the symmetry of combat models and their potential for conserved quantities derived for cases where separation of variables fails. 
J. Gill et al., Reconstructing Lanchester Warfare Models ...

\section{ACKNOWLEDGEMENT}

This project was conducted as part of DSTG's Summer Vacation Placement program.

\section{REFERENCES}

Casetta, L. (2015), 'The inverse problem of lagrangian mechanics for a non-material volume', Acta Mechanica 226(1), 1-15.

URL: https://doi.org/10.1007/s00707-014-1156-7

Darboux, G. (1878), 'Memoire sur l'approximation des fonctions de tres-grands nombres, et sur une classe etendue de developpements en serie', Journal de mathematiques pures et appliquees 3rd serie 4(4), 5-56.

Deitchman, S. (1962), 'A lanchester model of guerrilla warfare', Operations Research 6(10), 818-827.

Kress, M. \& MacKay, N. J. (2013), Bits or Shots in Combat? The Generalized Deitchman Model of Guerrilla Warfare.

Leubnert, C. \& Krumm, P. (1990), 'Lagrangians for simple systems with variable mass', European journal of physics 11(1), 31-34.

Mei, F. X., Wu, H. B. \& Zhang, Y. F. (2014), 'Symmetries and conserved quantities of constrained mechanical systems', International Journal of Dynamics and Control 2(3), 285-303.

URL: https://doi.org/10.1007/s40435-013-0043-8

Pincombe, A. H., Pincombe, B. M. \& Pearce, C. E. M. (2016), Dispersed combat as mass action with finite search, in M. Nelson, D. Mallet, B. Pincombe \& J. Bunder, eds, 'Proceedings of the 12th Biennial Engineering Mathematics and Applications Conference, EMAC-2015', Vol. 57 of ANZIAM J., pp. C305-C319. http://journal.austms.org.au/ojs/index.php/ANZIAMJ/article/ view/10447 [November 10, 2016].

Taylor, J. G. (1978), On Liouville's Normal Form for Lanchester-Type Equations of Modern Warfare with Variable Coefficients.

Taylor, J. G. (1981), 'Battle-outcome-prediction conditions for variable-coefficient lanchester-type equations for area fire', Journal of the Franklin Institute 311(3), 151-170.

Taylor, J. G. \& Brown, G. G. (1983), 'Annihilation prediction for lanchester-type models of modern warfare', Operations research 31(4), 752-771.

Yan, C. C. (1978), 'Construction of lagrangians and hamiltonians from the equation of motion', American journal of physics 46(6), 671-675. 